\title{
Influence of the human body mass in the open-air MRI on acoustic noise spectrum
}

\author{
Jiří Přibil ${ }^{1}$, Anna Přibilová ${ }^{2}$, Ivan Frollo ${ }^{1}$ \\ ${ }^{1}$ Institute of Measurement Science, Slovak Academy of Sciences, Bratislava, Slovakia \\ ${ }^{2}$ Institute of Electronics and Photonics, Faculty of Electrical Engineering \& Information Technology, Slovak University of Technology, \\ Bratislava, Slovakia
}

\section{ABSTRACT}

The paper analyses changes in spectral properties of the acoustic noise when the examined person lies in the scanning area of the open-air magnetic resonance imager (MRI). Consequently the holder of the lower gradient coils is loaded with the mechanical mass represented by the person's weight. The acoustic noise pressure level is mapped in the MRI neighborhood, too. Obtained results of spectral analysis will be used for the design of a correction filter to suppress the noise in the simultaneously recorded speech signal for 3D modeling of the human vocal tract.

Section: RESEARCH PAPER

Keywords: acoustic noise; noise reduction; signal processing; spectral analysis; statistical evaluation

Citation: Jiři Přibil, Anna Přibilová, Ivan Frollo, Influence of the human body mass in the open-air MRI on acoustic noise spectrum, Acta IMEKO, vol. 5, no. 3, article 13, November 2016, identifier: IMEKO-ACTA-05 (2016)-03-13

Editor: Paolo Carbone, University of Perugia, Italy

Received December 21, 2015; In final form May 26, 2016; Published September 2016

Copyright: (C) 2016 IMEKO. This is an open-access article distributed under the terms of the Creative Commons Attribution 3.0 License, which permits unrestricted use, distribution, and reproduction in any medium, provided the original author and source are credited

Funding: The work has been done in the framework of the COST Action IC 1206 and has been supported by the Grant Agency of the Slovak Academy of Sciences (VEGA 2/0013/14)

Corresponding author: Jiří Přibil, e-mail: jiri.pribil@savba.sk

\section{INTRODUCTION}

The magnetic resonance imaging (MRI) device usually consists of three gradient coils that produce three orthogonal linear fields for spatial encoding of a scanned object. From the physical principle follows that the rapid changes of the Lorentz forces during fast switching inside the weak static field $B_{0}$ environment [1] result in a significant mechanical vibration of these gradient coils. This process subsequently propagates in the air in the form of a progressive sound wave received by the human auditory system as a noise [2]. Due to its harmonic nature and the audio frequency range, the produced acoustic noise of this device can generally be treated as a voiced speech signal, and thus it can be recorded by a microphone and processed in the spectral domain using similar methods as those used for speech signal analysis.

The MRI technique enables analysis of the human vocal tract structure and its dynamic shaping during speech production while simultaneous recording of a speech signal [3], [4] is performed. The primary volume models of the human acoustic supra-glottal cavities constructed from the MR images can be transformed into three-dimensional (3D) finite element (FE) models [5]. Image and audio acquisition must be synchronized and the recorded speech signal must have a good signal-tonoise ratio so that high-quality results are achieved in $3 \mathrm{D}$ vocal tract model creation [6]. Several approaches to reduce the noise in the MRI equipment [7]-[9] are used in practice. One group of these enhancement methods is based on spectral subtraction of the estimated background noise when at least two microphones are used [10]. Our method uses only one microphone that picks up the noise of the running MRI scan sequence without phonation and then it records the speech signal during phonation with the background MRI noise. The following signal processing is carried out: The part of the signal containing only the MRI noise is used to calculate the basic spectral envelope using the mean Welch periodogram, and then it is analyzed in segments to determine the basic and supplementary spectral properties. The obtained spectral features are subsequently processed statistically and the 
achieved values are used to modify the basic spectral envelope of the noise signal that is further subtracted from the spectral envelope of the speech signal with the superimposed MRI noise. In general, these noise estimation techniques based on statistical approaches are not able to track real noise variations; thereby they result in an artificial residual fluctuation noise and a distorted speech. Therefore, the spectral properties of the acoustic noise generated by the gradient system of the MRI device must be analyzed with high precision so that the noise is efficiently suppressed while the maximum quality of the processed speech signal is preserved.

To investigate the transmission of vibration through the plastic holder of the MRI device scanning area, the measurement arrangement consists of the testing spherical water phantom which is useful for testing of the magnetic field homogeneity [11] in the device calibration phase. The situation changes when the examined person lies in the scanning area of the open-air MRI and the holder of the lower gradient coils is loaded with his/her weight. Then the mass of the whole mechanical system is altered and a change in spectral properties of the generated acoustic noise is expected, too. To verify this working hypothesis, the noise signal recording and its spectral analysis were performed for different person weights and with the water phantom only (for comparison). The obtained results will be used to devise an improvement of the developed cepstral-based noise reduction method [12] in the speech recorded during MRI scanning.

\section{ANALYSIS OF SPECTRAL PROPERTIES OF THE ACOUSTIC NOISE SIGNAL}

The spectrogram can be successfully used for visual comparison of differences in the time / frequency domain - see an example in Figure 1a,b. The disadvantage of subjectivity in this method can be eliminated by numerical matching of the spectral envelopes. To obtain the smoothed spectral envelope, the mean periodogram can be computed by the Welch method. In general, the periodogram represents an estimate of the power spectral density (PSD) of the input signal. Using the $N_{F F T}$-point FFT to compute the PSD as $S\left(e^{j \omega}\right) / f_{s}$ and the sampling frequency $f_{\mathrm{s}}$ in $\mathrm{Hz}$, we obtain the resulting spectral density in logarithmic scale expressed in $\mathrm{dB} / \mathrm{Hz}$ - see graphs in Figure 1c,d. The basic spectral properties can be determined from the spectral envelope and subsequently the histograms of spectral values can be calculated for objective matching - see Figure 1e.
Further detailed analysis in the frequency domain is done in the region of interest (ROI) - see the visualization in Figure 2. For numerical comparison it is possible to calculate the RMSbased spectral distances $D_{\text {RMS }}$ between periodograms corresponding to the MRI noise signals with different persons lying in the MRI device scanning area or using the sole phantom object. In addition, for evaluation of differences between spectral envelope values in the chosen frequency range, the absolute spectral difference $S_{\text {DIFF }}$ can be calculated by subtraction of their values in $\mathrm{dB}$. Analyzing this differential signal, the maximum value $\Delta P_{\max }$ can be localized and the corresponding frequency $F_{\max }$ can be determined - see the graphs (b) and (c) in Figure 2.

The supplementary spectral properties are usually determined from the frames (after segmentation and windowing). These properties describe the shape of the magnitude of the power spectrum $|S(k)|^{2}$ of the noise signal and they can be determined with the help of the additional statistical parameters [13]. The spectral centroid $\left(S_{\text {centr }}\right)$ is defined as a center of gravity of the spectrum, i. e. the average frequency weighted by the values of the normalized energy of each frequency component in the spectrum

$$
S_{\text {centr }}=\sum_{k=1}^{N_{\text {FFT }} / 2} k|S(k)|^{2} / \sum_{k=1}^{N_{\text {FET }} / 2}|S(k)|^{2}
$$

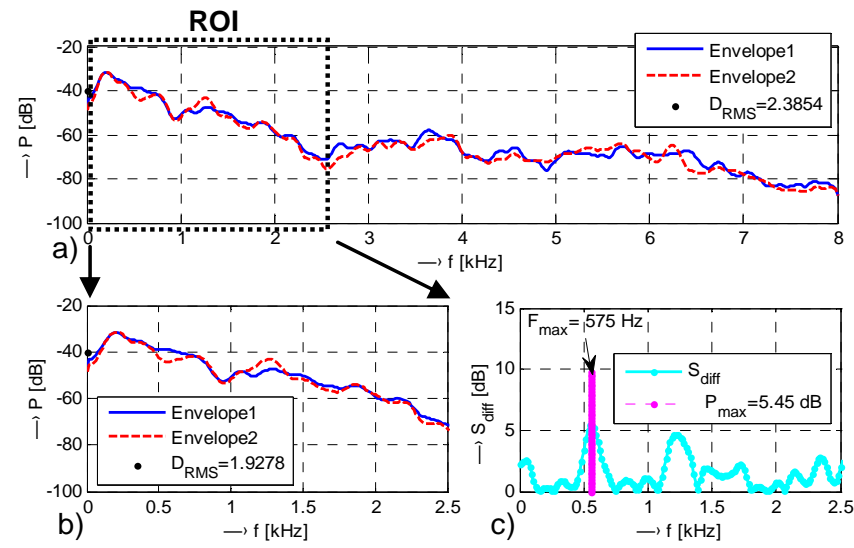

Figure 2. The smoothed envelopes by mean periodograms of two noise signals in the full frequency range $0 \sim f_{\mathrm{s}} / 2$ together with calculated RMS spectral distance (a), selected ROI in the low-frequency band $0 \sim 2.5 \mathrm{kHz}(\mathrm{b})$, depicted absolute differential signal $S_{\text {diff }}$ with localization of the frequency $F_{\max }$ corresponding to the maximum difference $\Delta P_{\max }$ (c).
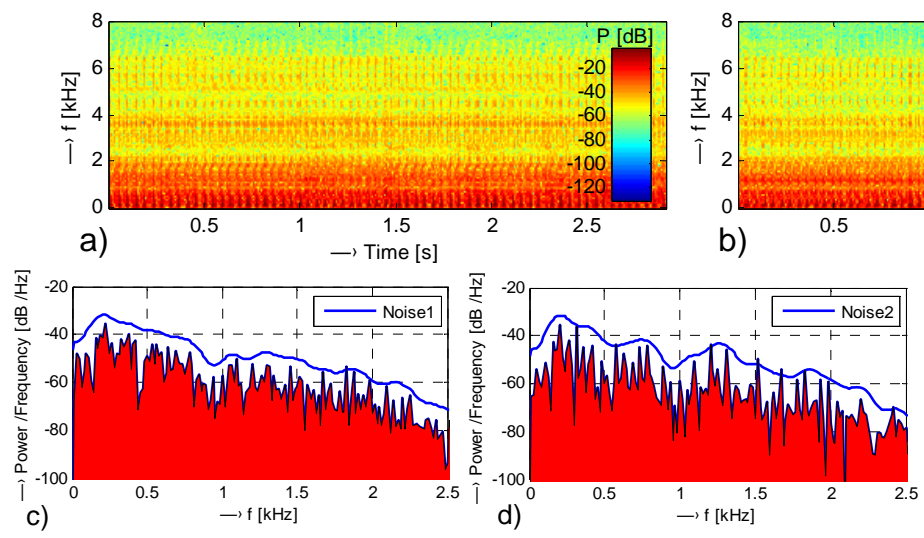

d)
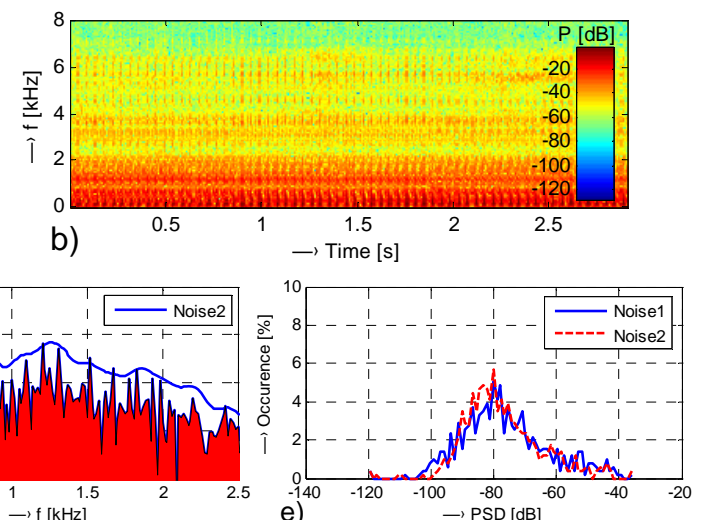

Figure 1. Spectrograms of two MRI noise signals (a, b), power spectral densities and their envelopes in the selected 250-ms ROIs depicted for the lowfrequencv band $0 \sim 2.5 \mathrm{kHz}(\mathrm{c})$-(d), histograms of these spectral envelopes (e). 
The spectral flatness $\left(S_{\text {flat }}\right)$ is determined as the ratio of the geometric and the arithmetic mean values of the power spectrum and it also describes the degree of periodicity in the signal [14]

$$
S_{f l a t}=\left[\prod_{k=1}^{N_{F F T}}|S(k)|^{2}\right]^{\frac{2}{N_{F F T}}} / \frac{2}{N_{F F T}} \sum_{k=1}^{N_{F F T}}|S(k)|^{2} .
$$

The spectral spread ( $\left.S_{\text {spread }}\right)$ parameter represents the dispersion of the power spectrum around its mean value

$$
S_{\text {spread }}=E(x-\mu)^{2}=\sigma^{2} \text {, }
$$

where $\mu$ is the first central moment and $\sigma$ is the standard deviation of the spectrum values. The spectral skewness $\left(S_{\text {skew }}\right)$ is a measure of the asymmetry of the data around the sample mean and can be determined as the third moment

$$
S_{\text {skew }}=\left(E(x-\mu)^{3} / \sigma^{3}\right) \text {. }
$$

The spectral kurtosis $\left(S_{\text {kurt }}\right)$ expressed by the fourth central moment represents a measure of peakedness or flatness of the shape of the spectrum relative to the normal distribution for which it is 3 (or 0 after subtraction of 3 )

$$
S_{\text {kurt }}=\left(E(x-\mu)^{4} / \sigma^{4}\right)-3 \text {. }
$$

\section{SUBJECT, EXPERIMENTS, AND RESULTS}

The analyzed open-air MRI equipment E-scan OPERA contains an adjustable bed which can be positioned in the range of 0 to 180 degrees, where the 0 degree represents the left corner near the temperature stabilizer device [15] - see the principal angle diagram of the MRI scanning area in Figure $3 \mathrm{~d}$. This noise has an almost constant sound pressure level (SPL) and consequently it can be easily subtracted as a background $\left(\mathrm{SPL}_{0}\right.$ ). Due to the low basic magnetic field $B_{0}$ (up to $0.2 \mathrm{~T}$ ) in the scanning area of this MRI machine, any interaction with the recording microphone must be eliminated. As the noise properties depend on the microphone position, the optimal recording parameters (the distance between the central point of the MRI scanning area and the microphone membrane, the direction angle, the working height, and the type of the microphone pickup pattern) must be found. The chosen type of the sequence together with the basic scan parameters repetition time (TR) and echo time (TE) - have significant influence on the scanning time. Values of these parameters result primarily from the chosen type of the scanning sequence. They can also be slightly changed manually but their final values depend on the setting of the other scan parameters - field of view (FOV), number of slices, slice thickness, etc.

The realization of the experiment with measurement of the acoustic noise produced by the gradient system of the MRI device consists of two phases. First, the noise signal is recorded by the pick-up microphone during execution of a scan MR sequence with different testing persons lying in the scanning area of the MRI device as documented by a photo in Figure 3a, and using only the testing spherical phantom with the diameter $14 \mathrm{~cm}$, filled with the solution of $\mathrm{CuSO}_{4}$ in a distilled water [15] $\left(\mathrm{CuSO}_{4}\right.$ shortens the TR time and speeds up the MR data collection) [11]. This phantom is placed in the middle point of the scanning area inside the scanning RF coil - see Figure $3 \mathrm{~b}$. Then, the noise signals are processed as follows:

1. Calculation of the basic and supplementary spectral properties of the recorded acoustic noise signals; determination of the main differences between the signals a)

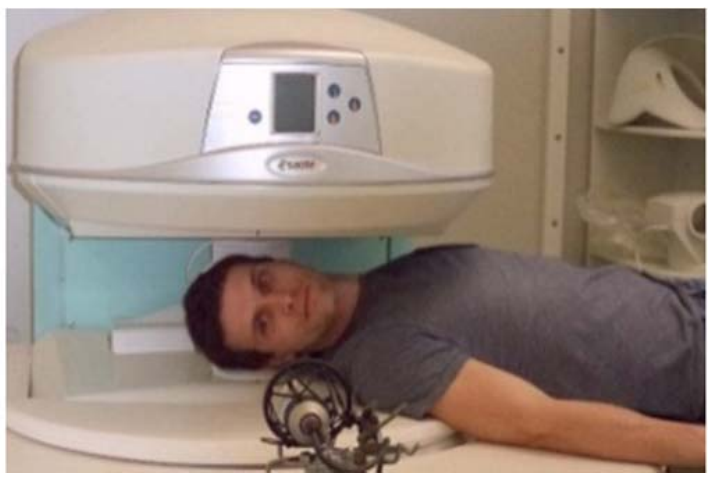

b)

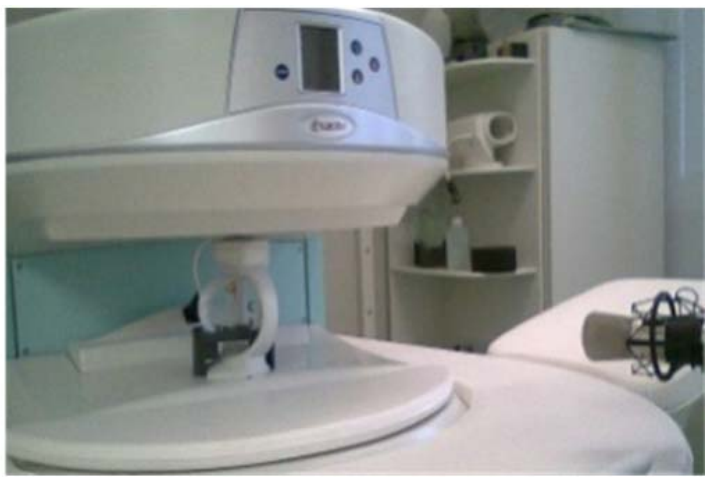

c)

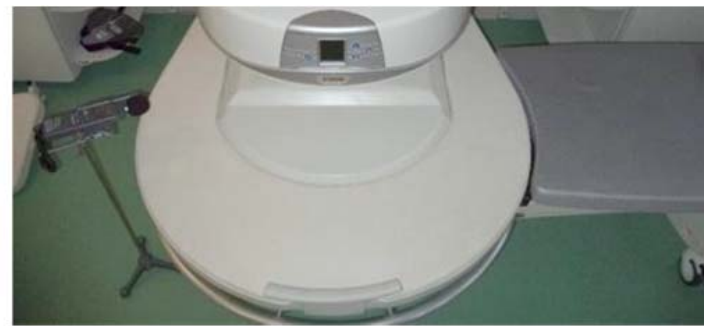

d)

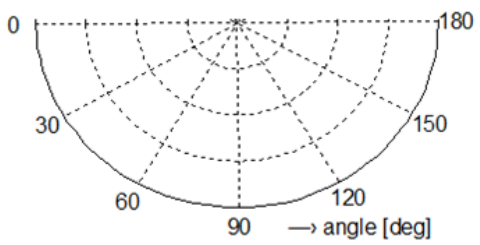

Figure 3. An arrangement of noise SPL measurement and recording in the MRI Opera: a lying person with a pick-up microphone at the position of 90 degrees (a), the testing water phantom with the recording microphone at the position of 150 degrees (b), the sound level meter at the position of 30 degrees (c), a principal angle diagram of the MRI scanning area (d).

with and without a lying person in the scanning area, and detailed analysis of the influence of different person weights on the spectral properties.

2. Visual comparison of the smoothed spectral envelopes in the full frequency range up to $f_{\mathrm{s}} / 2(0$ to $8 \mathrm{k})$, in the low frequency sub-band up to $2.5 \mathrm{kHz}$ ( 0 to $2 \mathrm{k}$ ), and the middle frequency sub-band of $2 \sim 6 \mathrm{kHz}$ (2 to $6 \mathrm{k}$ ); determination of the spectral distances $D_{\text {RMs }}$ of these envelopes between individual persons or the phantom object, and localization of the frequency $F_{\max }$ corresponding to the maximum difference $\Delta P_{\max }$ within the low-frequency band 0 to $2 \mathrm{k}$.

3. Statistical processing of determined values of the basic and supplementary spectral properties; numerical matching of the obtained results.

Comparison of spectral envelopes in the low-frequency band up to $2.5 \mathrm{kHz}$ together with their histograms for the selected 
pairs can be seen in Figure 4. The observed differences between the selected supplementary spectral properties are visualized by histograms in Figure 5, the summarized numerical values are introduced in Table 1.

The SSF-3D scanning sequence chosen for this comparison experiment can be used for the 3D MR scans of the human vocal tract [12], [16]. The auxiliary parameters of the used sequence were set as follows: $\mathrm{TE}=10 \mathrm{~ms}, \mathrm{TR}=45 \mathrm{~ms}, 10$ slices, 4-mm thick, sagittal orientation. The spherical testing phantom described earlier in this section was inserted in the scanning RF knee coil when the noise signal was recorded without a lying testing person in the MRI scanning area.

In correspondence with our previous research [12], the mapping of the acoustic noise SPL in the MRI neighborhood was performed for the mentioned scan sequence. The sound level meter of the multi-function environment meter Lafayette DT 8820 (with the range set to $35 \sim 100 \mathrm{~dB}$ ) was used for the measurement of the noise SPL at directions of 30, 90 and 150 degrees - see the obtained values in a graphical form in
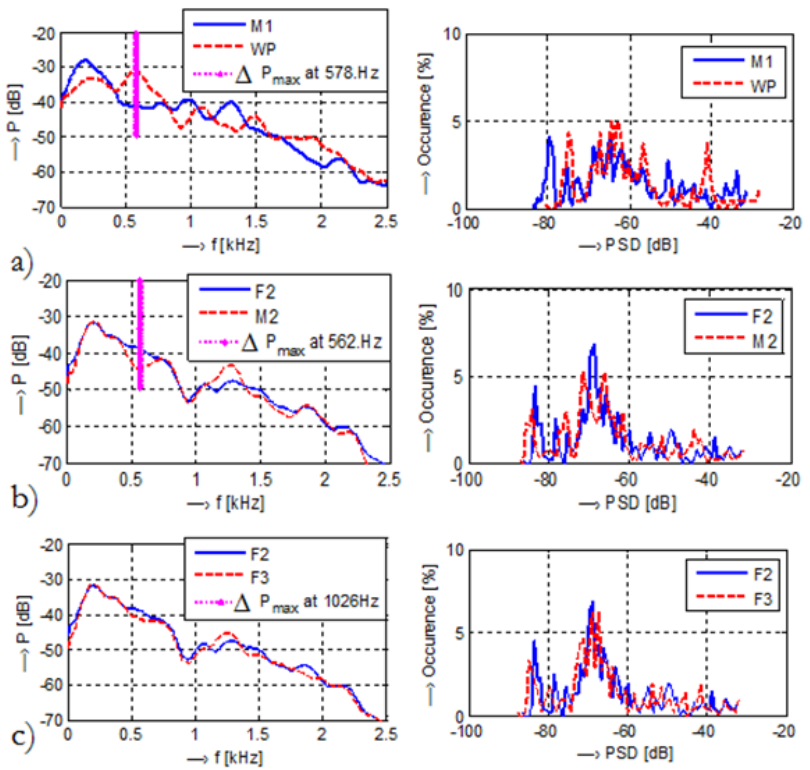

Figure 4. Spectral envelopes in the frequency band up to $2.5 \mathrm{kHz}$ and their histograms for the selected pairs: 78-kg male M1 / 0.75-kg phantom WP (a) 50-kg female F2 / 75-kg male M2 (b), 50-kg female F2 / 55-kg female F3 (c); microphone at 90 degrees.

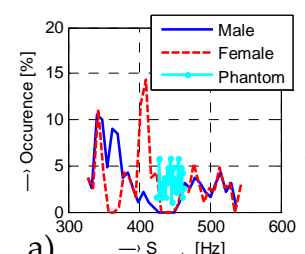

a)

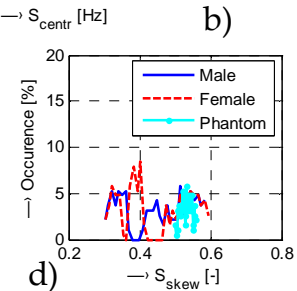

b) $\quad \stackrel{0.015}{\rightarrow} \mathrm{S}_{\text {flat }}[\mathrm{H}$
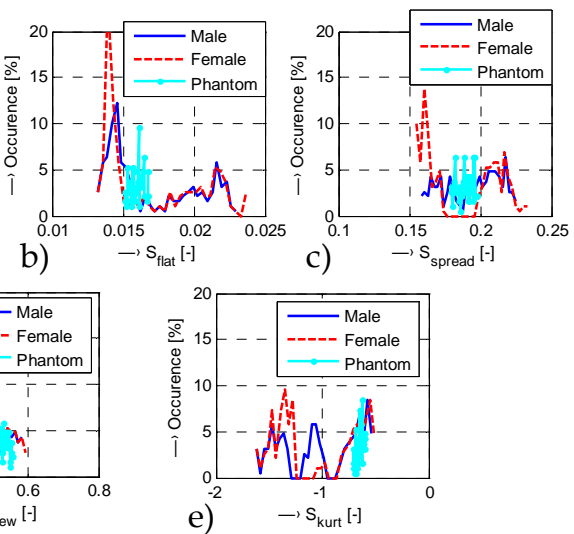

e) $\quad \rightarrow \mathrm{S}_{\text {kurt }}^{-2}[-]$

Figure 5. Histograms of the supplementary spectral properties: centroid (a), flatness (b), spread (c), skewness (d), and kurtosis (e) for the noise signals recorded at the position of 150 degrees.
Table 1. Summary comparison of mean values of spectral properties of the acoustic noise for tested pairs of male/female persons and water phantom (WP) inserted in the scanning area of the MRI device E-scan OPERA.

\begin{tabular}{cccccc}
\hline Tested pairs & $\begin{array}{c}\boldsymbol{F}_{\max } \\
{[\mathbf{k H z}]}\end{array}$ & $\begin{array}{c}\Delta \boldsymbol{P}_{\max } \\
{[\mathbf{d B}]}\end{array}$ & $\begin{array}{c}\boldsymbol{D}_{\text {RMs (0_2k) }} \\
{[\mathbf{d B}]}\end{array}$ & $\begin{array}{c}\boldsymbol{D}_{\text {RMs (2_6k) }} \\
{[\mathbf{d B}]}\end{array}$ & $\begin{array}{c}\boldsymbol{D}_{\text {RMs (0_8k) }} \\
{[\mathbf{d B}]}\end{array}$ \\
\hline Male/WP & 0.577 & 9.81 & 4.34 & 3.85 & 4.25 \\
Female/WP & 0.575 & 8.95 & 4.18 & 2.78 & 3.55 \\
Female/Male & 0.565 & 5.49 & 3.25 & 3.98 & 3.49 \\
Male/Male & 1.85 & 3.65 & 1.62 & 1.72 & 1.62 \\
Female/Female & 1.26 & 3.27 & 1.29 & 1.69 & 1.54 \\
\hline
\end{tabular}

Figure 6a. The SPL meter was located at the distances of 45, 60, and $75 \mathrm{~cm}$ from the central point of the scanning area (see Figure 3c). Subsequently the directional pattern of the acoustic noise SPL distribution was measured in the range of $<0-180>$ degrees, per 5 degrees, at the distance of $D_{\mathrm{L}}=60 \mathrm{~cm}$ from the MRI device central point with the inserted testing water phantom - see the resulting diagram including the $\mathrm{SPL}_{0}$ background values in Figure 6b.

The noise measurement was practically realized by real-time recoding of the signal by a microphone and transferring it to the external notebook during execution of a chosen scan MR sequence. The recording microphone was located at a distance of $60 \mathrm{~cm}$, at the horizontal positions of 30,90, and 150 degrees, and vertically in the middle between the both gradient coils. The input analogue noise signal, picked up by the 1 " Behringer dual diaphragm condenser microphone B-2 PRO with the cardioid polar pattern setting, was pre-amplified and processed by the mixer device Behringer XENYX 502 connected to the notebook by the USB audio interface U-CONTROL UCA202. The noise signal was recorded at a sampling frequency of $32 \mathrm{kHz}$, then resampled to $16 \mathrm{kHz}$, and subsequently processed. The stationary signal parts with a time duration of $8 \mathrm{~s}$ were selected and normalized to the level of $-16 \mathrm{~dB}$ using the sound editor program Sound Forge 8.0. The collected noise database originates from the records of six testing persons lying in the MRI scanning area: three males (M1-M3) with

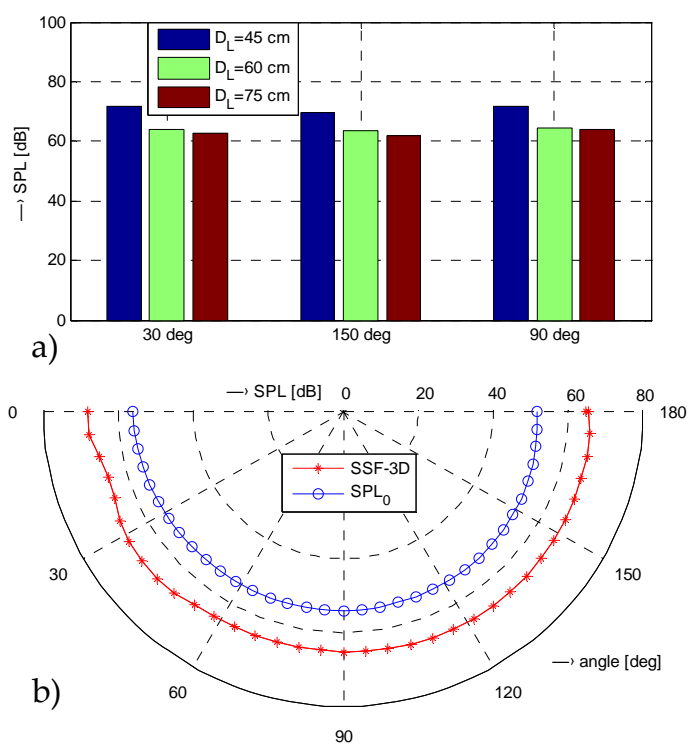

Figure 6. Bar-graph of the noise SPL values in the directions of 30,90, and 150 degrees at the distances of $D_{\mathrm{L}}=45,60$, and $75 \mathrm{~cm}(\mathrm{a})$, detailed directional pattern of the noise source together with the background noise $\mathrm{SPL}_{0}, D_{\mathrm{L}}=60 \mathrm{~cm}(\mathrm{~b})$ for the used SSF-3D MR scan sequence. 
approximate weights about $78 \mathrm{~kg}$, three females (F1-F3) with the mean weight of $53 \mathrm{~kg}$, and the testing water phantom with a holder (WP) weighting about $0.75 \mathrm{~kg}$. The patient's bed with an examined person was located at 180 degrees - this configuration was chosen since this has a minimal effect on the mentioned temperature stabilizer.

\section{DISCUSSION OF OBTAINED RESULTS}

The obtained results were evaluated by comparison of the basic spectral properties of the noise signals measured for the pairs with different weights: male/phantom, female/phantom, female/male, male/male, and female/female. The determined spectral differences of the noise signals between the persons with similar weights (female/female - see graphs in Figure 4c are small in all three observed frequency ranges. For the male/female pairs the spectral differences are greater and well visible in the spectral envelopes within the low-frequency band up to $2.5 \mathrm{kHz}$ - see the graphs in Figure $4 \mathrm{~b}$ (male person M3 with the weight of $80 \mathrm{~kg}$ ). For the male/phantom pairs the spectral differences are the greatest as can be noticed in the subplots of Figure $4 a$.

The achieved results of the supplementary spectral properties of the recoded noise mapped by histograms (see Figure 5) document that the range of values represented by the standard deviation is significantly broader for male and female persons than for the testing phantom. It was caused by the fact that the phantom of the same weight was used, but the values of both male and female groups were taken from the measurements using three persons of different weights. Next, the results of numerical matching of the noise spectral properties for the tested groups (phantom object, male or female persons) are in correlation with the graphical ones as shown by the summarized mean values in Table 2 .

Regarding the supplementary spectral properties of the recoded noise, the achieved results are in correlation with the obtained values of the distribution mapped by histograms (see Figure 5) and with numerical matching of the mean values of the noise spectral properties for the tested pairs summarized in Table 2. For small differences in the masses (similar weights of the subjects) the frequency of the detected maximum difference between the two spectral envelopes is higher than for the great differences between the masses (the weight of the male subject versus the water phantom giving this frequency about $570 \mathrm{~Hz}$ ).

From the performed comparison of the supplementary spectral properties for three tested locations of the recording microphone follows that no significant differences exist, however, the best results are obtained for the microphone position at 90 degrees, i. e. directed at the face of the lying person. At the microphone position of 30 degree the influence of the MRI temperature stabilizer can be superimposed as an

Table 2. Summary mean values of the supplementary spectral properties including the standard deviation values (in parentheses) of the analyzed noise signals summarized for all three recoding microphone positions.

\begin{tabular}{cccc}
\hline Property type & Male & Female & Phantom \\
\hline$S_{\text {centr }}[\mathrm{Hz}]$ & $406(70.8)$ & $425(71.9)$ & $485(10.9)$ \\
$S_{\text {flat }}[-]$ & $0.0145(0.0032)$ & $0.0138(0.011)$ & $0.0162(0.0005)$ \\
$S_{\text {spread }}[-]$ & $0.221(0.022)$ & $0.188(0.098)$ & $0.194(0.006)$ \\
$S_{\text {skew }}[-]$ & $0.531(0.093)$ & $0.452(0.373)$ & $0.629(0.016)$ \\
$S_{\text {kurt }}[-]$ & $-0.645(0.376)$ & $-1.017(1.728)$ & $-0.226(0.333)$ \\
\hline
\end{tabular}

additive noise with normal distribution. The microphone position at 150 degrees is unnatural from the point of the lying person and, in addition, the distance between the speaker face and the recording microphone was the longest.

\section{CONCLUSIONS}

The changes in the spectral properties of the noise signal generated by the gradient coils during the MRI scanning sequences while the examined person lay in the scanning area of the open-air MRI machine were analyzed. The resulting supplementary spectral features describe also the degree of voicing and the statistical properties of the noise component of the speech signal (type of noise, randomness, distribution, etc.). This information is necessary for correct application of the excitation in the cepstral speech reconstruction after noise suppression [12].

The obtained results will serve to create databases of initial parameters (such as the bank of noise signal pre-processing filters) for a developed cepstrum-based algorithm for noise suppression in the recorded speech. It will be useful in experimental practice, when it often occurs that the basic parameter setting of the used scanning sequence as well as the other scanning parameters must be changed depending on the currently tested person. In addition, it will be very interesting to carry out the detailed analysis and to determine the spectral properties of the MRI noise as a function of the mass of the subject. In this stage of our research only six volunteer persons (healthy people - colleagues) took part in our experiment, so it is very difficult or practically impossible. The solution to this issue may be cooperation with some medical centre (in Bratislava, Brno, etc.) having a certificate for the work with patients.

Finally, for better knowledge of the acoustic noise conditions in the scanning area and in the vicinity of the MRI device, accomplishment of additional measurement and experiments is necessary. To describe how the vibrations induce the acoustic noise and how they travel through the plastic holder of the MRI device, the time delay between the vibration impulses caused by the gradient coils and the noise signal must be analyzed, too.

\section{ACKNOWLEDGEMENT}

The authors would like to thank the volunteers from the Department of Imaging Methods, Institute of Measurement Science in Bratislava, for their help in our experiments.

\section{REFERENCES}

[1] A. Moelker, P.A. Wielopolski, M.T. Pattynama, Relationship between magnetic field strength and magnetic-resonance-related acoustic noise levels, Magnetic Resonance Materials in Physics, Biology and Medicine 16 (2003) pp. 52-55.

[2] G.Z. Yao, C.K. Mechefske, R.K. Brian, Acoustic noise simulation and measurement of a gradient insert in a $4 \mathrm{~T}$ MRI, Applied Acoustics 66 (2005) pp. 957-973.

[3] D. Aalto et al., Large scale data acquisition of simultaneous MRI and speech, Applied Acoustics 83 (2014) pp. 64-75.

[4] A.C. Freitas, M. Wylezinska, M.J. Birch, S.E. Petersen, M.E. Miquel, Comparison of Cartesian and non-Cartesian real-time MRI sequences at $1.5 \mathrm{~T}$ to assess velar motion and velopharyngeal closure during speech, PLoS ONE 11 (2016) e0153322. doi: 10.1371/journal.pone.0153322

[5] S.M.R. Ventura, D.R.S. Freitas, I.M.A.P. Ramos, J.M.T.S. Tavares, Morphologic differences in vocal tract resonance 
cavities of voice professionals: An MRI-based study, Journal of Voice 27 (2013) pp. 132-140.

[6] T. Vampola, A.M. Laukkanen, J. Horáček, J.G. Švec, Vocal tract changes caused by phonation into a tube: A case study using computer tomography and finite element modelling, of the Acoustical Society of America 129 (2011) pp. 310-315.

[7] G. Kannan, A.A. Milani, I.M.S. Panahi, R.W. Briggs, An efficient feedback active noise control algorithm based on reduced-order linear predictive modeling of fMRI acoustic noise, IEEE Transactions on Biomedical Engineering 53 (2011) pp. 33033309.

[8] D. Aalto et al., Large scale data acquisition of simultaneous MRI and speech, Applied Acoustics 83 (2014) pp. 64-75.

[9] X. Shou, et al., The suppression of selected acoustic frequencies in MRI, Applied Acoustics 71 (2010) pp. 191-200.

[10] G. Sun, et al., Adaptive speech enhancement using directional microphone in a 4-T scanner, Magnetic Resonance Materials in Physics, Biology and Medicine 28 (2015) pp. 473-484.
[11] I. Frollo et al., Measurement and imaging of planar electromagnetic phantoms based on NMR imaging methods, Measurement Science Review 10 (2010) pp. 97-101.

[12] J. Přibil, J. Horáček, P. Horák, Two methods of mechanical noise reduction of recorded speech during phonation in an MRI device, Measurement Science Review 11 (2011) pp. 92-98.

[13] Y. Dodge The Concise Encyclopedia of Statistics, Springer, 2008, ISBN 978-0-387-32833-1.

[14] S. Aleinik, O. Kudashev, "Estimating stochasticity of acoustic signals", in: Speech and Computer, LNCS 8773. A.Ronzhin, R.Potapova, V.Delic (editors). Springer, Cham, Heidelberg, New York, ISBN 978-3-319-11580-1, pp. 192-199.

[15] E-scan Opera, Image Quality and Sequences Manual, 830023522 Rev. A, Esaote, Genova, Italy, 2008.

[16] J. Přibil, D. Gogola, T. Dermek, I. Frollo, Design, realization, and experiments with a new RF head probe coil for human vocal tract imaging in an NMR device, Measurement Science Review 12 (2012) pp. 98-103. 